\title{
Editorial
}

\section{Type 1 diabetes: a genetic Pandora's box?}

In this issue of the journal, Delli et al. (1) describe the changing incidence of type 1 diabetes mellitus (T1DM) in children born in Sweden to immigrant parents, a topic of considerable scientific interest, since these changes must reflect environmental, rather than purely genetic influences. The take home message of this article (1) is that patients with T1DM born to immigrants in Sweden, a country that has a notoriously high incidence of T1DM, more frequently carry human leukocyte antigen (HLA)-DQ2 genotypes and possess glutamic acid decarboxylase 65 (GAD65) autoantibodies when compared to indigenous Swedish T1DM patients. Although this observation confirms that the effect of HLA-DQ2 is not neutral as initially thought (2), these results strongly argue that in the subset of T1DM patients described, additional genetic and/or environmental risk factors must contribute to the progression of overt disease (1). A recent genomewide association study (GWAS) and meta-analysis found over 40 loci that affect risk of T1DM (3). It is conceivable that several of those 40 loci identified by GWAS contain genes of possible functional relevance to the subgroup of T1DM individuals identified by Delli et al. (1). These loci include the region 1q32.1 which contains the potent immunoregulatory cytokine genes, IL-10, IL-19 and IL-20.

The interplay between the environment and genetic disposition is unquestionably complicated. It is proposed that the incidence of T1DM is increasing, with the diagnosis occurring more in younger age children (4). Although it is acknowledged that there may be an effect due to migration, the earlier age that children with T1DM are now presenting could be related to environmental changes (4). Zung et al. noted that in Jewish Ethiopians who are genetically at risk and who immigrated from Ethiopia to Israel (an area with a high incidence of T1DM), had an increased risk of developing T1DM as they adopted their new country's lifestyle (5). This contradicts the study by Ehehalt et al. which found that Italian immigrant children had incidence rates of T1DM that were similar to their home country and not their new country of residence, therefore reducing the importance of the environmental influence (6).
The study by Delli et al. (1) expands the findings of Ehehalt's study(6) by investigating whether multigenerational origin influenced children's risk of developing T1DM in an area that has a high incidence of this disease. In particular, parents and grandparents were identified as either Swedish (all family members), non-Swedish or of mixed origins (some family members of Swedish descent). HLA typing and islet autoantibody testing were then performed on those younger than 18 years who were diagnosed with T1DM. At least one of the HLA genotypes, either DQ2 or DQ8, was found in $80 \%$ of these patients, a frequency significantly higher than that found in children of Swedish descent. In $85 \%$ of the patients, at least one islet autoantibody was confirmed with almost half having two or more autoantibodies. Islet autoantibodies tended to be more frequent in those of Swedish descent, although not statistically significant. When the investigators examined if specific HLA markers were associated with specific autoantibodies, they found that the HLA-DQ2 haplotype, more frequent in immigrant parents, was associated with the GAD65 antibody, whereas the HLA-DQ8 HLA haplotype, more frequent in those of Swedish descent, was associated with IA-2 autoantibodies. In those with neither DQ2 or DQ8, non-Swedish patients had increased levels of autoantibodies, which led the authors to postulate that there are other genes leading to autoimmunity (1).

It is suggested that different ethnic groups have unique genetic predispositions to developing T1DM. Kawasaki et al. reported on a form of diabetes that they termed 'fulminant' T1DM, a subgroup of T1DM in Japanese patients, in whom there are no autoantibodies and who do not possess the known susceptibility HLA haplotypes (7). This leads us to inquire more about the patients without autoantibodies in the study by Delli et al. (1). Were their HLA haplotypes similar to expectations or did they vary from those usually associated with T1DM, suggesting that perhaps the risk of developing diabetes is not always HLA-dependent? In addition, only three autoantibodies were checked in this study, so it is possible that those without detected autoantibodies could still have distinct autoantibodies that have yet to be identified (8). The origin of the 
non-Swedish patients (immigrants from the Middle East and North Africa) could also be relevant given that it is reported that $\sim 1 / 2$ of African-Americans with new-onset T1DM are negative for antibodies at diagnosis (9). Finally, it was unclear how long the children had lived in Sweden. Is it possible that those without antibodies had not lived in Sweden a sufficient amount of time to develop autoimmunity?

In summary, this study contributes important information about how immigration may affect a child's risk of developing T1DM in an area that has a known high incidence of this disease. However, more investigations are needed to determine whether new risk alleles influence disease susceptibility and pathogenesis in the ethnic groups with and without evidence for islet autoimmunity identified by Delli et al. (1).

Inas $H$ Thomas, $M D,{ }^{a}$ and Massimo Pietropaolo, $M D^{b}$

${ }^{\text {a }}$ Department of Pediatrics and Communicable Diseases (Division of Pediatric Endocrinology), University of Michigan Medical School, Ann Arbor, MI 48109, ${ }^{\mathrm{b}}$ Laboratory of Immunogenetics, The Brehm Center for Diabetes Research, Department of Internal Medicine (Division of Metabolism, Endocrinology and Diabetes), University of Michigan Medical School, Ann Arbor, MI 48109 e-mails: inash@med.umich.edu; maxtp@med.umich.edu

\section{References}

1. Delli AJ, Lindblad B, Carlsson A, et al. Type 1 diabetes patients born to immigrants to Sweden increase their native diabetes risk and differ from Swedish patients in HLA types and islet autoantibodies. Pediatric Diabetes 2010: 11: 513-520.

2. Resic-Lindehammer S, Larsson K, Ortqvist E, et al. Temporal trends of HLA genotype frequencies of type 1 diabetes patients in Sweden from 1986 to 2005 suggest altered risk. Acta Diabetol 2008: 45: 231-235.

3. Barrett JC, Clayton DG, Concannon P, et al. Genome-wide association study and meta-analysis find that over 40 loci affect risk of type 1 diabetes. Nat Genet 2009: 41: 703-707.

4. Ehehalt S, Dietz K, Willasch AM, Neu A, Baden-Wuerttemberg Daibet I. Epidemiological perspectives on type 1 diabetes in childhood and adolescence in Germany - 20 years of the Baden-Wurttemberg Diabetes Incidence Registry (DIARY). Diabetes Care 2010: 33: $338-340$.

5. Zung A, Elizur M, Weintrob N, et al. Type I diabetes in Jewish Ethiopian immigrants in Israel: HLA class II immunogenetics and contribution of new environment. Hum Immunol 2004: 65: 1463-1468.

6. Ehehalt S, Popovic P, Muntoni S, et al. Incidence of diabetes mellitus among children of Italian migrants substantiates the role of genetic factors in the pathogenesis of type 1 diabetes. Eur J Pediatr 2009: 168: 613-617.

7. Kawasaki E, Eguchi K. Is type 1 diabetes in the Japanese population the same as among Caucasians? Ann NY Acad Sci 2004: 1037: 96-103.

8. Pietropaolo M, Becker DJ, Laporte Re, et al. Progression to insulin-requiring diabetes in seronegative prediabetic subjects: the role of two HLA-DQ high-risk haplotypes. Diabetologia 2002: 45: 66-76.

9. Winter WE, Maclaren NK, Riley WJ, Clarke DW, KaPpy MS, SPILlar RP. Maturity-onset diabetes of youth in Black-Americans. N Engl J Med 1987: 316: 285-291. 